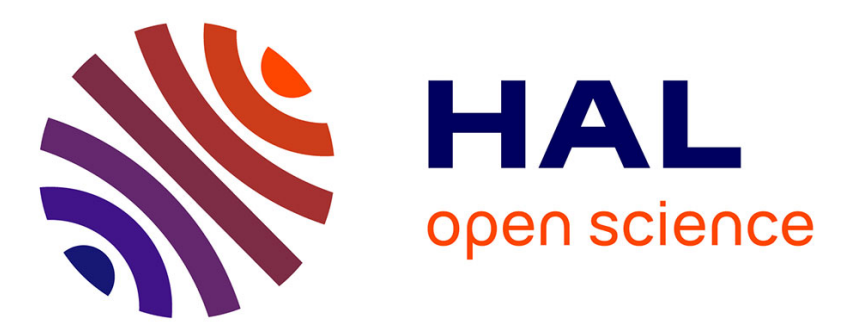

\title{
Aménagement moderne des forêts dans une région protégée. Problèmes actuels et futurs du Parc national des Abruzzes en Italie
}

Lucia Naviglio, Francesco Spada, Franco Tassi

\section{- To cite this version:}

Lucia Naviglio, Francesco Spada, Franco Tassi. Aménagement moderne des forêts dans une région protégée. Problèmes actuels et futurs du Parc national des Abruzzes en Italie. Revue forestière française, 1977, 29 (6), pp.476-483. 10.4267/2042/21171 . hal-03396398

\section{HAL Id: hal-03396398 \\ https://hal.science/hal-03396398}

Submitted on 22 Oct 2021

HAL is a multi-disciplinary open access archive for the deposit and dissemination of scientific research documents, whether they are published or not. The documents may come from teaching and research institutions in France or abroad, or from public or private research centers.
L'archive ouverte pluridisciplinaire HAL, est destinée au dépôt et à la diffusion de documents scientifiques de niveau recherche, publiés ou non, émanant des établissements d'enseignement et de recherche français ou étrangers, des laboratoires publics ou privés. 


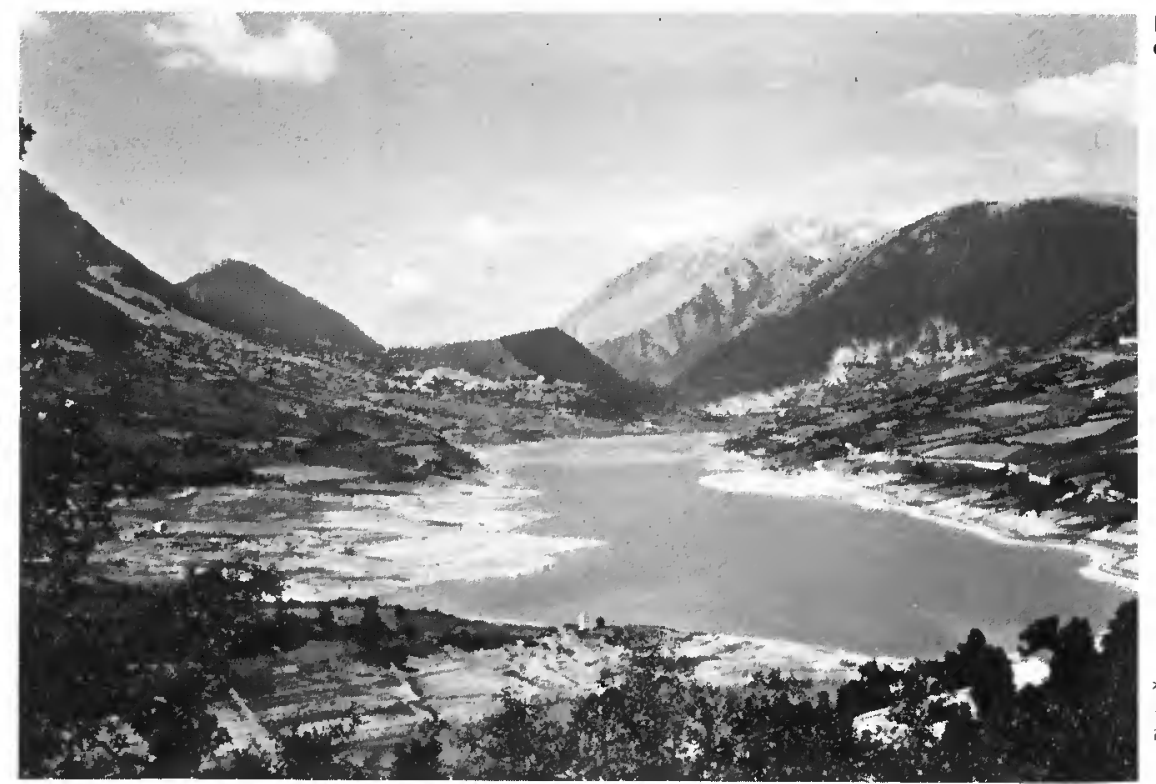

La "Camosciara» : le cœur du parc Hext 3

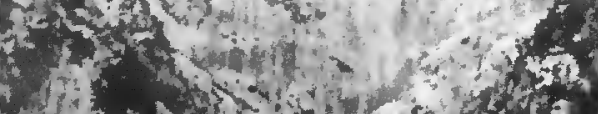

(f)

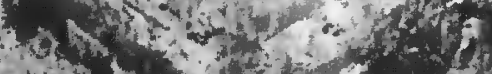

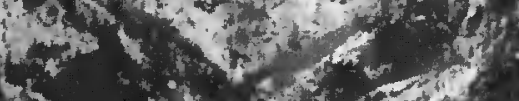

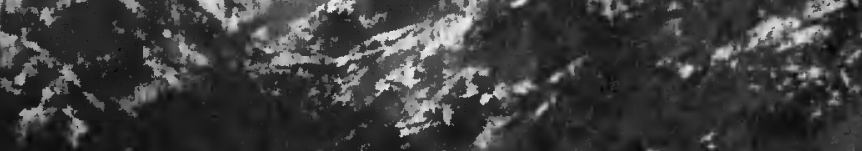

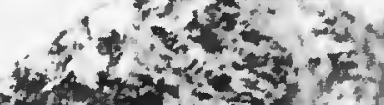

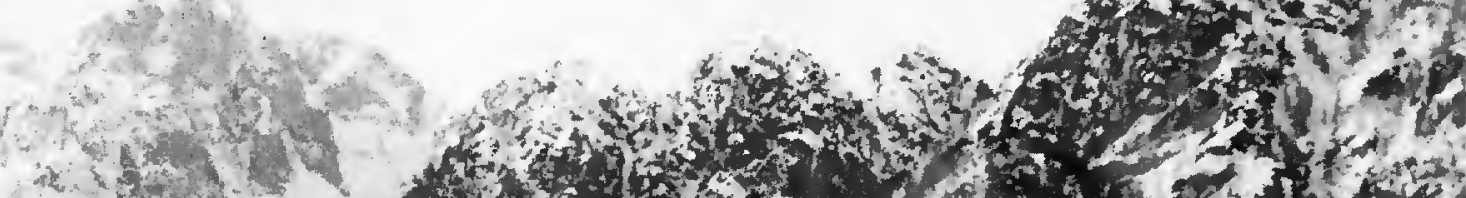

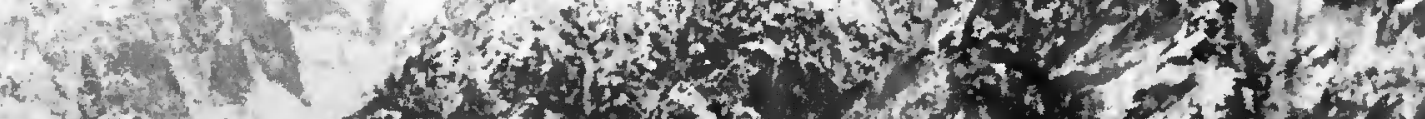

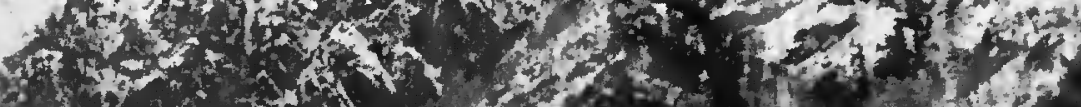

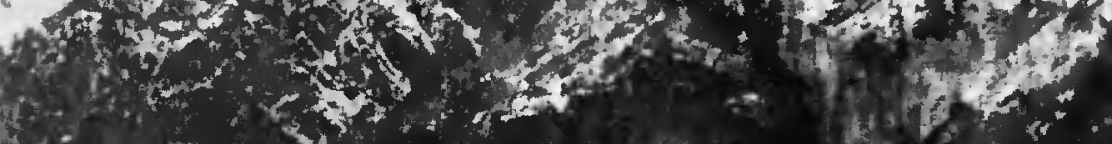
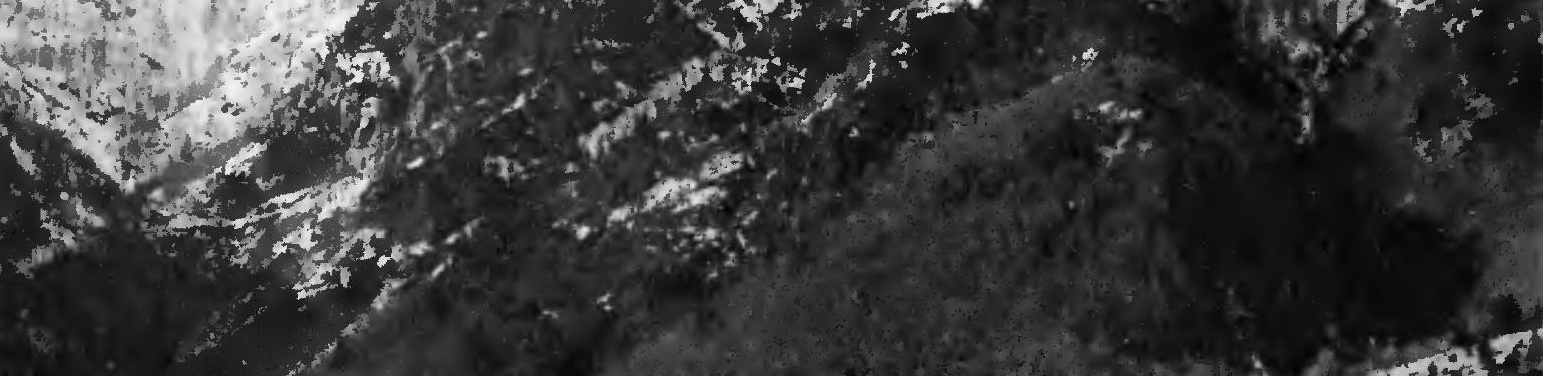
Cette rubrique est dirigée par

$$
\text { J.-P. LARRIVAL }
$$

Ingénieur du G.R.E.F.

Ministère de l'Agriculture

Service des Forêts

1 ter, avenue de Lowendal 75007 PARIS

\title{
AMÉNAGEMENT MODERNE DES FORÊTS DANS UNE RÉGION PROTÉGÉE
}

\author{
Problèmes actuels et futurs \\ du Parc national des Abruzzes en Italie
}

\author{
L. NAVIGLIO - F. SPADA - F. TASSI
}

Class. Oxford $62: 907.11$ (45)

Le problème de la gestion des écosystèmes et par conséquent des forêts dans les Parcs nationaux n'est pas un problème simple, mais il se pose de façon urgente dans de nombreux parcs. Aussi a-t-il semblé intéressant de faire connaitre à nos lecteurs le type de réflexions que se posent des collègues italiens et les solutions qu'ils proposent.

Même si, plus ou moins consciemment, ils considèrent la production comme une "dimension primaire » de la forêt, on s'apercevra, en lisant leur article, que l'analyse fine préalable et nécessaire les conduit nécessairement à de nouvelles vues sur l'aménagement global des formations boisées du Parc national des Abruzzes, et par suite à un certain nombre de tensions et de propositions dont bien entendu ils gardent, comme il est de règle dans notre Revue, toute la responsabilité.

N.D.L.R.

Les fonctions d'une forêt, surtout dans une région protégée, sont actuellement bien plus diverses et complexes qu'auparavant. Dans les milieux culturels et scientifiques on attribue, en effet, à la forêt une dimension multiple $\left({ }^{1}\right)$ : primaire ou de production, secondaire ou de sauvegarde, tertiaire ou de récréation et quaternaire ou de recherche scientifique. On voudrait, en effet, ne plus considérer la forêt dans son interprétation classique, mais plutôt l'écosystème

(1) On ne considère pas seulement la production de bois, mais aussi celle de tous les produits de la forêt, faune comprise; la sauvegarde n'a pas seulement un caractére hydrogéologique, mais elle se veut aussi oxigénation, filtrage de l'aérosol, etc.: la récréation sera considérée comme une éducation, une culture, un équilibre psycophysique, etc. 


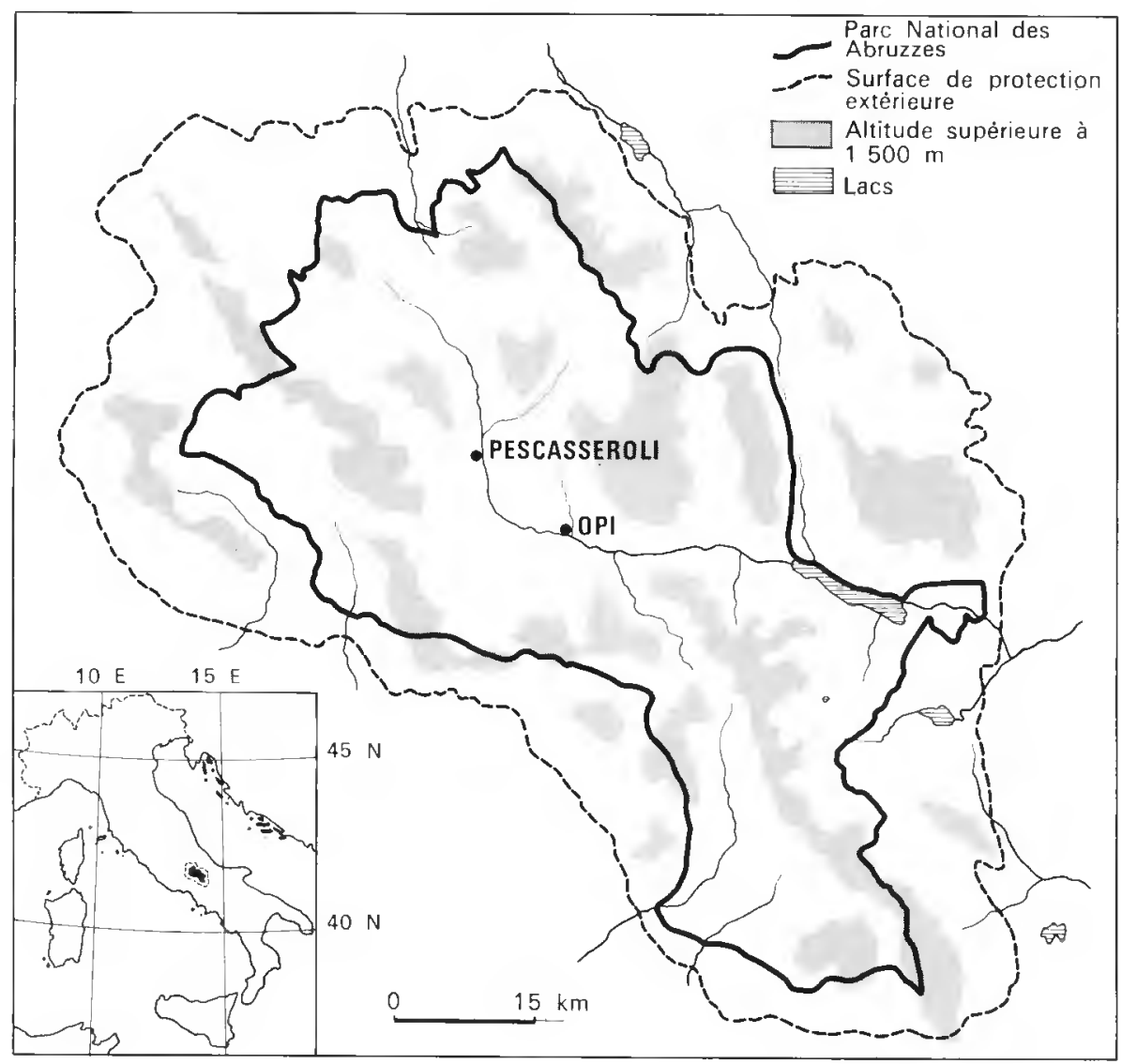

forestier dans son ensemble. Le passage d'une mentalité et d'une technique étroitement "sylviculturelles", économiques et de production à des modèles de protection efficaces est très souvent difficile et discuté. Nous allons décrire l'histoire et les perspectives de reconversion en cours, dans un parc national européen parmi les plus intéressants par son écosystème forestier et sa faune : le Parc national des Abruzzes, dans l'Italie centrale.

Le territoire du Parc (40000 ha) est couvert de forêts pour 61,5\% (24600 ha), de pâturages pour $31,4 \%$ et de champs cultivés pour 7,5\%. La plupart des forêts (de 1000 à $1800-1900 \mathrm{~m}$ d'altitude) sont composées de hêtre (Fagus silvatica). Aux expositions plus chaudes, aux altitudes inférieures, il est remplacé par Quercus cerris et Ostrya carpinifolia.

Le Parc national des Abruzzes héberge de nombreuses espèces végétales et animales fort intéressantes; une grande partie d'entre elles sont des reliques glaciaires.

Parmi les animaux, il faut avant tout nommer le chamois qui est représenté ici par une sousespèce particulière, Rupicapra rupicapra ornata et qui est considéré comme le chamois le plus beau du monde; l'ours aussi, représenté par une centaine d'exemplaires, est une sousespèce endémique. Ursus arctos marsicanus; le loup. Canis lupus italicus, est très rare et en danger du fait de la chasse, bien qu'il soit légalement protégé. Parmi les oiseaux les plus intéressants, il faut rappeler l'aigle royal qui niche dans le Parc, les chocards et les craves à bec rouge, le pic de Lilford (Dendrocopus leucotus liffordi), le hibou grand-duc. Nommons encore la loutre, le chat sauvage, l'écureuil qui est ici presque noir avec la poitrine blanc pur, 
la vipère d'Ursini, espèce très localisée dans les hautes terres des Apennins et de nombreux animaux plus ou moins répandus dans les autres parties de l'Italie ou de l'Europe. De plus, dès les premières années de la réorganisation du Parc (à partir de 1969), la réintroduction de deux espèces d'ongulés disparus auparavant, le cerf rouge et le chevreuil, a été effectuée avec beaucoup de succès.

Les forêts du Parc ont une histoire très complexe, car elles ont toujours été fortement et de façon très irrégulière exploitées par l'homme qui en a modifié la physionomie d'une façon particulière en rapport avec l'activité humaine prédominante.

Jusqu'aux années 1800, il y avait dans la région du Parc une activité agro-pastorale très poussée $\left({ }^{2}\right)$; on défrichait les bois dans les vallées jusqu'à $1300 \mathrm{~m}$, afin d'étendre les champs cultivés et on éliminait en altitude la partie supérieure de la forêt, sur une bande de $200 \mathrm{~m}$ au moins, pour étendre les pâturages.

Les animaux pâturaient aussi sous bois, y dégradaient le sol et empêchaient la régénération naturelle. En outre, des clairières nouvelles étaient continuellement créées et agrandies par le feu.

Aujourd'hui, on peut encore observer des exemples de bois pâturés et de pré-bois aux hêtres typiquement écimés. Le développement de l'activité pastorale eut son apogée en 1600 , après quoi on enregistra un déclin jusqu'au début du XXe siècle quand l'évolution économique incita les habitants de l'Apennin central à abandonner la montagne. A ce moment-là, les bois et les sols étaient dégradés et il y avait des vides nombreux ; une partie des forêts était constituée par des taillis mal traités depuis longtemps, tandis qu'une autre partie était formée de futaies irrégulières. Les forêts encore épargnées par les coupes restaient toutefois assez vastes, car situées dans les lieux peu accessibles où se cachait encore une faune des plus intéressantes. Les forêts avaient souffert du développement désordonné de l'activité pastorale ainsi que de l'exploitation des bois pour la satisfaction des exigences les plus variées. Parmi celles-ci, certaines devaient se maintenir bien que peu rentables, telle la production de la "scandola " (tuile en bois pour les toits) pour laquelle furent utilisés les hêtres les mieux formés, ou la fabrication de tous les ustensiles en bois utilisés pour les besoins domestiques et sylvopastoraux. Entre 1895 et 1911, l'intense activité pastorale fut remplacée par une exploitation des forêts toujours plus importante. Dans la période 1922-1932, les coupes représentaient un volume de $2314 \mathrm{~m}^{3} /$ an et elles augmentèrent jusqu'à $7423 \mathrm{~m}^{3} /$ an entre 1945 et 1954 . Mais au lieu des " coupes irrégulièrement décidées » des années précédentes, on assista à la conversion des taillis en futaie, et des structures en général irrégulières on passa souvent à des structures équiennes. Entre 1945-1954, dans le Parc, on coupa en moyenne 1,99 $\mathrm{m}^{3} / \mathrm{ha} / \mathrm{an}$ pour un accroissement moyen annuel de $1,97 \mathrm{~m}^{3}$. Entre 1910 et 1939 , seulement 3 des 18 communes du territoire du Parc avaient des aménagements pour l'exploitation de leurs bois et un seulement restait valable pour la période 1969-1978. On estime que jusqu'à 1969, année de l'établissement de la nouvelle direction du Parc, 40000 à 50000 hêtres étaient coupés annuellement - et environ 600.000 à 700000 avaient été abattus pendant la période 1955-1967 dans le Parc - pour fournir l'argent nécessaire aux budgets des communes. Pourtant, on n'a jamais trouvé les résultats concrets de ces revenus dans les réalisations publiques de ces autorités locales! Même l'autorité forestière a dû admettre que ces données sous-estiment fortement la situation réelle, bien qu'elle n'ait jamais voulu faire connaitre publiquement les chiffres officiels. Entre 1961 et 1969, on a encore coupé des arbres, en partie même abusivement, notamment pour l'implantation d'installations destinées aux sports d'hiver.

La situation actuelle est la suivante : $96 \%$ du territoire boisé appartient aux communes et $4 \%$ aux propriétaires privés. $10 \%$ des bois sont des futaies jardinées; $25 \%$ sont équiennes ;

(2) En 1881, il y avait environ 52000 ovins, mais en 1960 ils étaient déjả tombés à presque 7000 seulement. 
$45 \%$ sont des futaies irrégulières (intermédiaires entre les deux catégories précédentes); $10 \%$ des bois proviennent d'anciens pâturages et $10 \%$ demeurent en taillis. Dans les lieux les plus inaccessibles, survivent encore de très petits ténements (peut-être 5 ha) de forêts vierges, très intéressants en tant que modèle pour l'étude de la conversion. Certains bois sont très beaux, ont une régénération naturelle abondante et possèdent des arbres très grands (hauteur de plus de $30 \mathrm{~m}$ et diamètre de plus d'un mètre : on peut compter jusqu'à 420 cernes d'accroissement annuel).

Actuellement, la politique nouvelle du Parc national autorise dans certaines conditions et en certains endroits, avec des garanties de sauvegarde tout à fait spéciales, des coupes de bois de chauffage pour les paysans, mais il est toujours interdit de couper les grands arbres. Pour sensibiliser l'opinion publique et assurer la survie de ces monuments exceptionnels de nos forêts, le Parc a lancé, en coopération avec le World Wildlife Fund, une «Opération Grand Arbre ". A l'avenir, pour satisfaire les exigences écologiques et de protection "sensu lato", on aménagera les forêts d'une façon tout à fait nouvelle. Pour commencer, et préalablement à tout nouvel aménagement, on a réalisé des études floristiques, phytosociologiques et de productivité primaire.

La présence inévitable de la pression humaine sur une partie des forêts du Parc (où il n'est pas toujours possible de donner des indemnités pour permettre l'interdiction des coupes) rend nécessaire l'adoption d'une exploitation tolérable avec la conservation de la biocœnose forestière, structure et composition floristique comprises. II faut d'abord individualiser des " modèles » vers lesquels diriger l'évolution spontanée de la végétation en place après les prélèvements antérieurs en forêt. II faut encore choisir des interventions sylvicoles qui permettent d'atteindre les modèles floristiques et structuraux préalablement définis dans des temps pas trop longs. La situation actuelle des forêts du Parc des Abruzzes dérive directement de l'action humaine qui a donné aux cénoses une structure et une composition particulières. La formation des taillis jadis, puis leur conversion en futaie, a créé un type de forêt avec une flore et une structure qui ne correspondent pas toujours, au point de vue naturaliste, aux finalités multiples d'une forêt dans une région protégée : cas, par exemple, dans une hêtraie, de la transformation des taillis en futaies. On peut actuellement identifier floristiquement dans le Parc national :

10 une hêtraie caractérisée par la présence de houx (llex aquifolium) qui occupait probablement jadis une zone plus grande à la limite inférieure de l'actuelle distribution du hêtre ;

$2^{\circ}$ des restes très localisés indiquant que probablement il y avait au-dessus de la zone 1 une bande phytoclimatique de Fagus-Taxus, aujourd'hui presque détruite par les bergers qui éliminaient les ifs ;

30 la plupart des forêts étaient des hêtraies dont la physionomie floristique était caractérisée par l'absence des étages de végétation arborescente subordonnée au hêtre, avec une flore herbacée rare et souvent absente et une structure plus simple qu'en zones 1 et 2 . En somme :

a) le facies actuel dérive de la dégradation par l'homme des forêts à Fagus-l/ex ou Fagus-//ex-Taxus après l'élimination des strates arborescentes subordonnées (les coupes de taillis ont conduit à une temporaire "continentalisation » du microclimat forestier, défavorable à /lex et Taxus ici à la limite climatique de leur distribution):

b) la structure équienne à laquelle on a conduit les futaies à partir du taillis, ne permet plus le développement d'espèces telles que Acer, Cerasus, Sorbus, typiques des hêtraies à plus haute altitude:

c) cette pauvreté en flore et structure est typique des hêtraies plus évoluées au point de vue sylvicole, mais elle traduit une situation de collapsus floristique qui empêche une régénération naturelle et oblige la faune à abandonner de vastes régions de forêts où elle ne trouve rien à manger. 
Dans l'esprit d'une restauration du milieu à partir des forêts exploitables, on aboutira à une nouvellc complication floristique des phytocénoses forestières en modifiant la structure actuelle de la futaie en régénération. L'étude de la végétation potentielle nous dira exactement quelle doit être l'extension des types Fagus-//ex et Fagus-//ex-Taxus.

II faut aussi adopter le modèle Fagus-Acer-Rosaceae où l'analyse phytosociologique et épionthologique nous indique l'ancienne existence d'une hêtraie pure (Fagetea filicinea sensu Domin). En effet, Acer et Rosacea ont une fonction pionnière qui assure une superposition et une alternance (chronologique plus que structurelle) entre l'étage dominant du hêtre et l'étage dominé. Aujourd'hui, pour former la base d'une reconversion "naturelle " de l'écosystème "forêt exploitable », on peut seulement "démailler » par des coupes irrégulières la couverture trop épaisse des houppiers des futaies de hêtre pour favoriser le développement des phytocénoses subordonnées et la structure irrégulière à âges variés. II faut surtout préférer la suppression des hêtres qui vivent à côté d'autres espèces, pour favoriser la venue d'éléments nouveaux (cas typique des cénoses Fagus-Acer à dominance alternée). De fortes éclaircies permettraient aussi une régénération plus importante du hêtre. II faut également augmenter les centres naturels d'extension des espèces autres que le hêtre, en considérant toujours leur distribution potentielle. Ces dispositions impliquent le rétablissement des vieilles techniques d'abattage des arbres, ce qui ne devrait pas poser un grave problème du fait de la présence dans le Parc national des Abruzzes d'infrastructures classiques de culture traditionnelle encore valable dans le contexte local.

Les études sur la productivité primaire ont été conduites dans le cadre du Programme biologique international, section productivité terrestre, dans le but de rechercher le type structurel ayant la productivité majeure. On a commencé par l'étude de la production de la litière qui contribue à la formation de l'humus et à l'équilibre des cycles bio-géochimiques des éléments minéraux. La futaie qui a donné la plus grande quantité de litière est celle qui a une structure irrégulière, bien qu'elle ait un pourcentage de feuilles mangées par les insectes plus grand que les futaies régulières et équiennes (tableau n 1). La hêtraie "Difesa " a donné une quantité de litière statistiquement différente des autres.

Tableau $n^{\circ} 1$

\section{Caractéristique des trois hêtraies en cours d'étude \\ (moyenne sur 4 ans)}

\begin{tabular}{|c|c|c|c|c|c|c|}
\hline Hêtraie & $\begin{array}{c}\text { Nombre } \\
\text { d'arbres } \\
\text { par ha }\end{array}$ & $\begin{array}{c}\text { Type de bois } \\
\text { Futaie }\end{array}$ & $\begin{array}{c}\text { Circonférence } \\
\text { moyenne } \\
(\mathrm{cm})\end{array}$ & $\begin{array}{c}\text { Hauteur } \\
\text { moyenne } \\
(\mathrm{m})\end{array}$ & $\begin{array}{c}\text { Litière } \\
\text { feuilles } \\
(\mathrm{t})\end{array}$ & $\begin{array}{c}\% \text { surface } \\
\text { feuilles } \\
\text { mangées }\end{array}$ \\
\hline Val Fondillo . & 438 & Régulière & 102 & 23 & 3,1 & 4 \\
Difesa . . . & 268 & Irrégulière & 102 & 14 & 3,4 & 13,2 \\
Bisegna . . . & 441 & Régulière & 86 & 16 & 3,2 & 11,1 \\
\hline
\end{tabular}

Dans le cadre de la nouvelle politique du Parc national, on a dépensé pour la sauvegarde des forêts (et de certains pâturages et vides importants) de 1969 à 1976 une somme d'environ 300 millions de lires italiennes, en ayant le contrôle effectif de la situation sur 8000 ha, soit presque $1 / 5$ du territoire du Parc.

En particulier, on a déjà pris en location presque 6300 ha de forêts et on a empêché des coupes déjà autorisées par le Service forestier sur 785 ha, en payant en contrepartie 163,5 millions de lires, et en obtenant ainsi la protection d'environ 130000 hêtres de dimensions 
moyennes. Un cas particulier est celui des bois qui se trouvent dans les 10000 ha récemment entrés dans le Parc national : on a pu y sauver 10000 hêtres. II faut que les coupes soient interdites surtout sur les stations les plus intéressantes; les tractations nécessaires sont en cours.

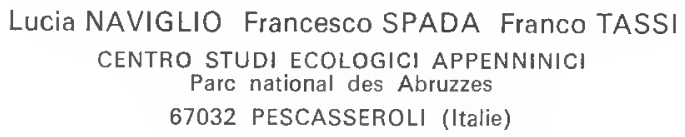

\section{BIBLIOGRAPHIE}

BORTOLOTTi (L.). - Contributo alla difesa del paesaggio del Parco nazionale d'Abruzzo. - Monti e Boschi, Bologna, vol. 19, n², 1968, pp. 6-16.

BORTOLOTTI (L.). - Documenti sul Parco Nazionale d'Abruzzo. Collana verde, no 23, 1969, pp. 1-114.

BORTOLOTTI (L.). - Le faggete nel paesaggio del Parco Nazionale d'Abruzzo. Quaderni del Parco Nazionale d'Abruzzo, no 4, 1972, pp. 69-7B.

BRUNO (F.), BAZZICHELI (G.). - Note illustrative alla carta della vegetazione del Parco Nazionale d'Abruzzo. Progetto conservazionale geobotanico. Annali di Botanica, vol. 28, n 3, 1966, pp. 739-778.

CLAUSER (F.). - Un interessante itinerario forestale nel Parco Nazionale d'Abruzzo. Monti e Boschi, no 9, 1958, pp. 501-506.

CLAUSER (F.). - Boschi ed economia forestale nel Parco Nazionale d'Abruzzo, Collana Verde, no 14, 1964 , pp. $1-83$

CRIVELLARI (D.). - Conservazione e miglioramento delle taggete alpine ed appenniniche. Atti del Congresso nazionale di Selvicoltura per il miglioramento e la conservazione dei boschi italiani, Firenze, 14-18 marzo 1954, 1955, pp. 237-2B4.

GENTILE (S.). - Sui faggeti dell'italia meridionale. Atti dell'/stituto Botanico e Laboratorio Crittogamico delI'Università di Pavia, vol. 6, no 5, 1969, pp. 207-306.

ITALIA NOSTRA. - Piano di riassetto del Parco Nazionale d'Abruzzo. - Roma, Italia nostra, 1968. - $259 \mathrm{p}$.

NAVIGLIO (L.). - Researches on beech-forest ecosystems. III. Description of the three beech forests studied in the Abruzzo National Park (Monti Marsicani, Abruzzo). Annali di Botanica, 1975.

PATRONE (G.). - Assestamento e conversione dei cedui composti. Pubblicazioni della regia Università di Firenze, Facoltà di Scienze Agrarie e Forestali, vol. 18, 1940, pp. 1-96.

RUBEL (E.). - Die Buchenwaldes Europas. Veroffentlichungen des geobotanischen Institutes der E.T.H. Zurich, 1932, 509 p.

SIMONETTA (A.M.), BRUNO (F.), COLACICCHI (R.), BORTOLOTTI (L.). - Piano naturalistico del Parco. Quaderni del Parco Nazionale d'Abruzzo, no 2, 1971, pp. 1-61.

TASSI (F.). - Idee per la natura. Quaderni del World Wildlife Fund, no 1, 1970, pp. 1-70.

TASSI (F.). - Problemi di conservazione dell'ecosistema forestale nei Parchi Nazionali. Informatore botanico italiano, vol. 2, no 3, 1970, pp. 169-177.

VISONA (L.), NAVIGLIO (L.). - Researches on beech forest ecosystems. IV. Litter production on the Mount Terminillo IBP Station (Monti Reatini, Lazio) and in three beech forests in the Abruzzo National Park (Monti Marsicani, Abruzzo) in 1972-1973. Annali di Botanica, 1975.

VISONA (L.), NAVIGLIO (L.). - Researches on beech forest ecosystems. V. The influence of phytophagous insects on leaf litter production at Mount Terminillo IBP Station (Monti Reatini, Lazio) and in three beech forests of Abruzzo National Park (Monti Marsicani, Abruzzo) in 1972. Annali di Botanica, 1975. 